\title{
An Assessment of Zebrafish Natural Color Preference and its Modification by Stimuli
}

\section{Dawn Salmon and Mohammad Kutub Ali*}

\author{
Department of Basic Medical Sciences, University of the West Indies, Jamaica
}

\begin{abstract}
Zebrafish have been known to show color preference. Conditions that enhance or diminish color preferences are largely inconclusive. In this study, we used the T-Maze with red and green arms to test the Zebrafish color preference. Our results indicated that the initial exposure of individually housed starved fish to the T-maze produced four types of swimming patterns with no indication of a clear preference for a particular colored arm. However, this situation changes with subsequent exposure to the T-maze, as the fish started to develop a clear color preference for green than red. In contrast, starved fish removed individually from their shoaling environment and exposed to the T-maze showed no biased color preference between green and red, and no food seeking activity. Starved shoaling fish exposed to the T-maze in groups, had a greater preference for green than red and displayed an increase in exploration of the red arm as well as increased feeding activity within the red arm. Well-fed shoaling fish displayed the same behavior, but had a lower tendency of exploring the non-preferred color arm (red). Addictive drugs modified the behavior of the well-fed shoaling fish; however modification decreased with increasing tolerance against the addictive drugs. Their color preference for green than red can be effectively used to understand the diverse mode of action of addictive drugs and cross-talk. For example, pre-treatment of well-fed shoaling fish with alcohol and nicotine singly, produced increased and decreased preferences respectively for green compared to the control without affecting their preference for red. In contrast, alcohol-nicotine pretreated fish, initially displayed the alcohol effect, but nicotine overtook the alcohol effect with increasing time after drug withdrawal from co-treatment. Overall our results clearly suggest that Zebrafish have a greater color preference for green than red which can be modified by different stimuli, but cannot be eliminated.
\end{abstract}

Keywords

Zebrafish, Color preference, T-Maze, Alcohol, Nicotine, Percentage Distribution of Fish (PDF)

Abbreviations

PDF $=$ Percentage Distribution of Fish

\section{Introduction}

Zebrafish (Danio rerio) is a very versatile and suitable vertebrate model which is widely used to study hyperactivity and anxiety $[1,2]$, appetite $[3,4]$ and associative learning and memory $[5,6]$. Zebrafish possess all the sensory processes such as taste, tactile, smell, balance and hearing. Their excellent color vision is one of the most widely studied sensory systems [7]. It has been reported that Zebrafish decision-making is highly influenced by their natural color preference [8-10]. However, Zebrafish natural color preference has been highly debated because of many contradictory conclusions. For example, it has been reported that Zebrafish have no natural bias toward a particular color, but instead, their color preference can be largely influenced by learning or after using training paradigms [11]. Similar studies were carried out by two different research groups with different outcomes. One reported that Zebrafish have no color bias toward red or green [12], while the other reported more preference for blue and green compared to yellow and red [7]. Both of these studies were contrary to previous studies which

*Corresponding author: Mohammad Kutub Ali, Department of Basic Medical Sciences, University of the West Indies, Mona, Kingston-7, Jamaica, Tel: 1-(876)-826-2166, E-mail: alikutub@yahoo.com

Received: February 11, 2017; Accepted: May 15, 2017; Published online: May 17, 2017

Citation: Salmon D, Ali MK (2017) An Assessment of Zebrafish Natural Color Preference and its Modification by Stimuli. J Mood Disord Ther 1(1):17-23

Copyright: (c) 2017 Salmon D, et al. This is an open-access article distributed under the terms of the Creative Commons Attribution License, which permits unrestricted use, distribution, and reproduction in any medium, provided the original author and source are credited. 


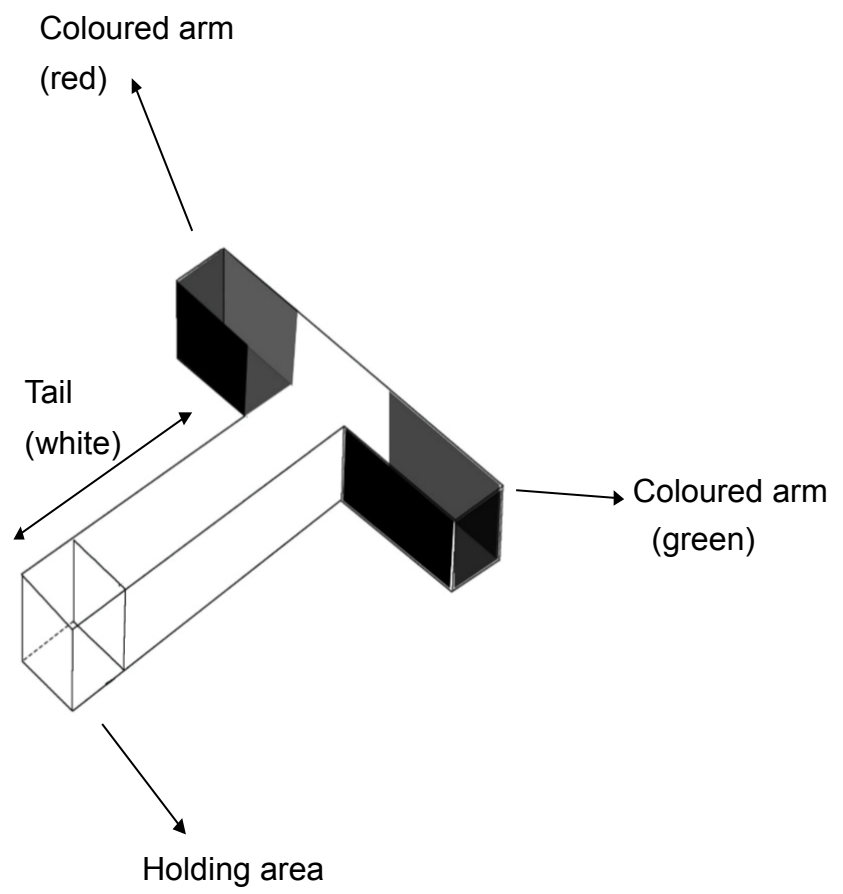

Figure 1: Schematic model of color arms of T-maze tank having measurements of both green and red arm: $31.75 \mathrm{~cm}$ $\times 25.4 \mathrm{~cm} \times 25.4 \mathrm{~cm}$; holding area: $16.51 \mathrm{~cm} \times 25.4 \mathrm{~cm} \times$ $25.4 \mathrm{~cm}$; tail: $76.2 \mathrm{~cm} \times 25.4 \mathrm{~cm} \times 25.4 \mathrm{~cm}$.

suggested Zebrafish have a strong color preference towards red that can be modified but note liminated by training [13]. Thus, whether Zebrafish have a natural color preference or not, is still inconclusive. The color preference of Zebrafish under the influence of chemical stimulants such as addictive drugs has not been fully explored. Therefore we have evaluated the effect of alcohol and nicotine, two of the most widely used stimulants, on Zebrafish color preference. Earlier studies carried out, have found that zebrafish which are exposed to alcohol display changes in behaviors such as aggression, locomotion, stress and startle responses while nicotine caused a reduction of stress and enhanced learning [14]. But, recent studies suggest that alcohol and nicotine might share the same neuronal nicotinic Acetylcholine Receptors (nAChRs) [15]. Many investigators have been working to develop a suitable alcohol-nicotine co abuse animal mode to reveal the molecular mechanism, but only a few have been endorsed [16]. Therefore it would be interesting to investigate how both alcohol and nicotine individually and in combination influence Zebrafish color preference. In this study, we have re-evaluated the Zebrafish color preference using a T-Maze tank consisting of a red arm, green arm and white tail under various conditions. Our studies, unlike the others previous reported, showed that Zebrafish have a strong color preference towards green which can be modified momentarily by different stimulating factors but not eliminated.

\section{Methodology}

Normal healthy adult Zebrafish which were housed under a normal light dark (14/10 hr) cycle were selected for this study. The selected fish were maintained separately in two groups for 10 days. In one group, fish were maintained individually [1 fish/1.5 L; tank size (L) 21 $\times(\mathrm{W}) 11 \times(\mathrm{H}) 5.5 \mathrm{~cm}$ ] or in a group of six (denoted as shoaling group) $[6 \mathrm{fish} / 6 \mathrm{~L}$; tank size $(\mathrm{L}) 30 \times(\mathrm{W}) 20 \times$ $(\mathrm{H}) 17 \mathrm{~cm})]$ under optimum conditions of water and temperature. No reduction in performance was reported even when fish housing conducted with 12 fish/1L [17]. The fish were fed twice daily with blood worms obtained from Aqua Select, Taiwan. The study of fish swimming pattern were carried out within 30 minutes of their release into T-maze tank (Figure 1) having green arm, red arm and white tail. The images were captured with a CCD camera fitted above the tanks. All recording were carried out in a closed room to ensure no external influence and disturbance took place during the recording. The tracking and analysis of recorded images were carried out by NIH Image J software and statistical analysis were conducted using Microsoft Excel single factor ANOVA.

\section{Results and Discussion}

\section{Experiment set I}

Screening of different types of swimming pattern of individually housed starved Zebrafish in the T-maze tank (initial exposure). Four types of swimming patterns were generated from the analysis of swimming pattern, for overnight starved fish, when no food source was present inside the tank. Out of the 30 individual fish studied, 12 fish showed type 1 preferential swimming shuttle between the white tail and green arm (Figure 2A); 6 fish showed type 2 between white tail and red arm (Figure 2B). 8 fish showed type 3 preferential shuttling between white tail, green and red arms (Figure 2C) and 4 fish showed type 4 i.e., shuttling within white tail zone with no significant movements towards either green or red arms or both (Figure 2D). Type 1 and type 2 were classified based on the fish overall retention time in each arm i.e., greater than 5 minutes in green arm (type 1) or red arm (type 2) during an entire 30 minutes of recording. Type 3 was classified when the fish overall retention time in both the green and red arm were more than 10 minutes (i.e., more than 5 minutes in each arm). Type 4 was assigned when the overall retention time in both the green and red arm was less than 10 minutes (i.e., time spent in each arm was less than 5 minutes) during the entire 30 minutes of recording.

\section{Experiment set II}

Screening of swimming pattern of individually housed starved Zebrafish after subsequent exposure to the T-maze. 6 starved fish which either went to the green or red arm 
Citation: Salmon D, Ali MK (2017) An Assessment of Zebrafish Natural Color Preference and its Modification by Stimuli. J Mood Disord Ther 1(1):17-23

\section{Top View}

\section{Type 1 \\ Type 2 \\ Type 3 \\ Type 4}

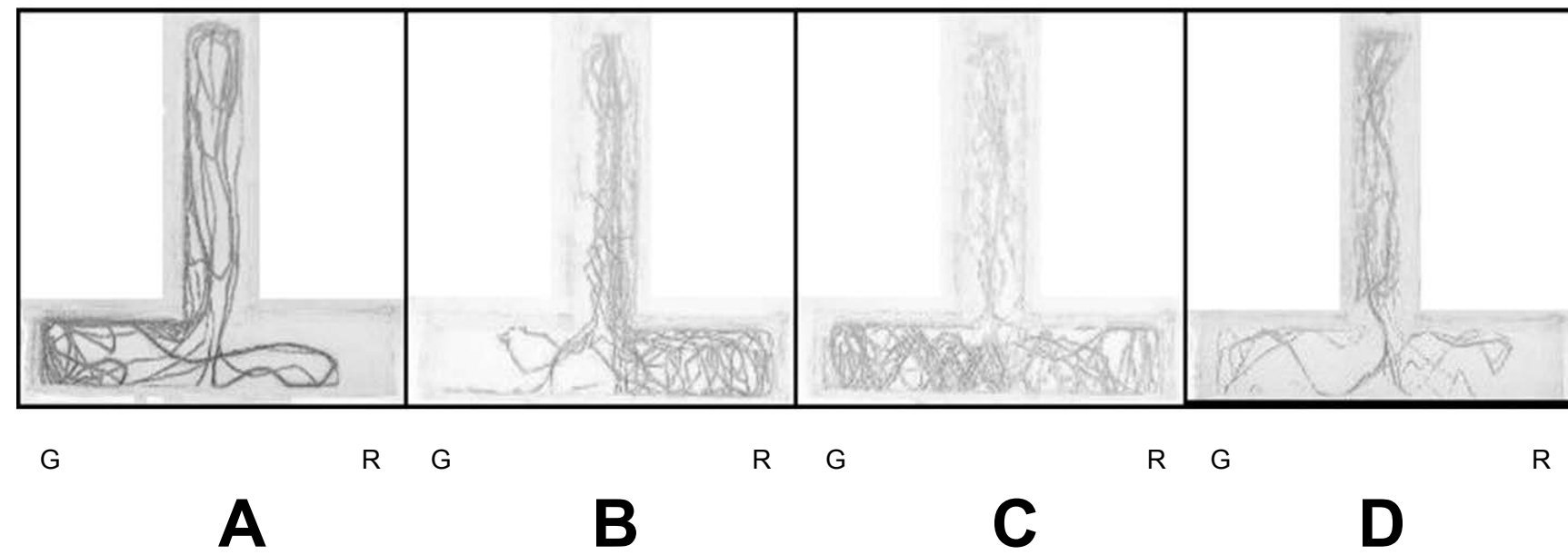

Figure 2: Representative image of different types of swimming patterns observed on the first day of exposure by the individually housed starved Zebrafish in the T-maze tank during 30 minutes of recording ( $G=$ Green; $R=R e d)$.

Table 1: Total percentage distribution of individually housed starved Zebrafish in each colored arm of the T-maze tank during 30 minutes of recording.

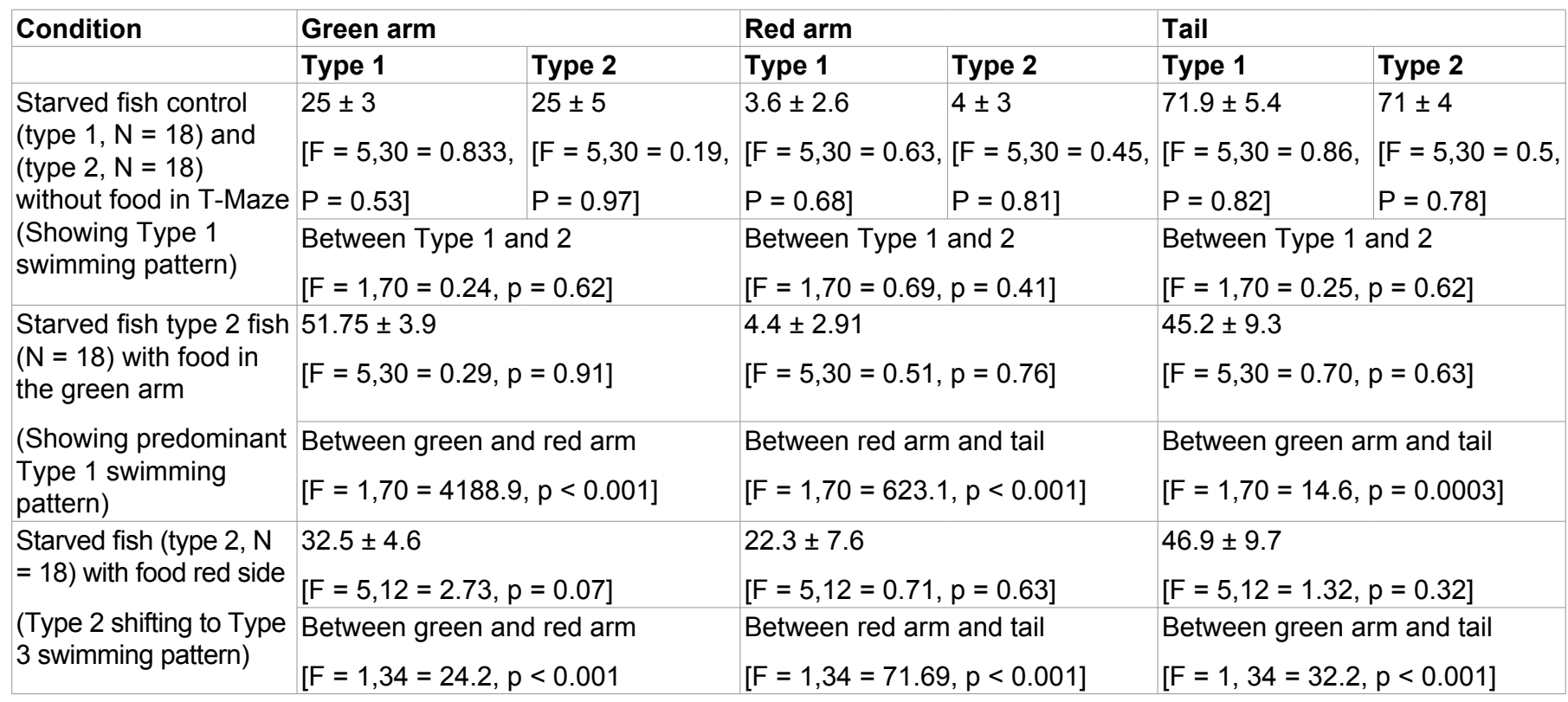

(Value expressed as Mean \pm SD of 6 consecutive recording with 6 fish of type 1 and 2 in triplicate; Different treatment groups have different set of 6 fish of each type in triplicate).

during their first day exposure were selected. Fish were exposed to the T-maze for an additional five days. The experiments were repeated twice with a new set of fish selection (as carried out in experiment set one), bringing each type of fish population to 18 . Surprisingly, type 1 retained its swimming pattern while type 2 shifted to type 1 swimming pattern (Table 1, Row 1). This shifting from type 2 swimming pattern to type 1 swimming pattern as well as their retention time in the green arm drastically increased when the food was introduced into the green arm (Table 1, Row 2). In contrast, when the food was in- troduced into the red arm, the fish which displayed type 1 swimming pattern did not shift to type 2 swimming pattern (data not shown). However, fish which displayed type 2 (three replicates, each with 6 fish of the type) swimming pattern showed only marginal improvement in their retention time in the red arm. Further, swimming pattern completely shifted from type 2 to type 3 (Table 1, Row 3). Since fish were not trained to choose either the green or red arm, based on their observed behavior, it can be suggested that Zebrafish have a strong color preference toward green compared to red color. In- 
crease in retention time when the food was in the green arm, and only a marginal increase in retention time in the red side, directly correlated to their color preference. There was a clear indication that the red color induced some degree of anxiety in the fish, as there was a reduc- tion in their food seeking activity in that arm, in addition to shifting of their swimming pattern from type 2 to type 1 (in the absence of food in the red arm); type 2 to type 3 (in presence of food in red arm).
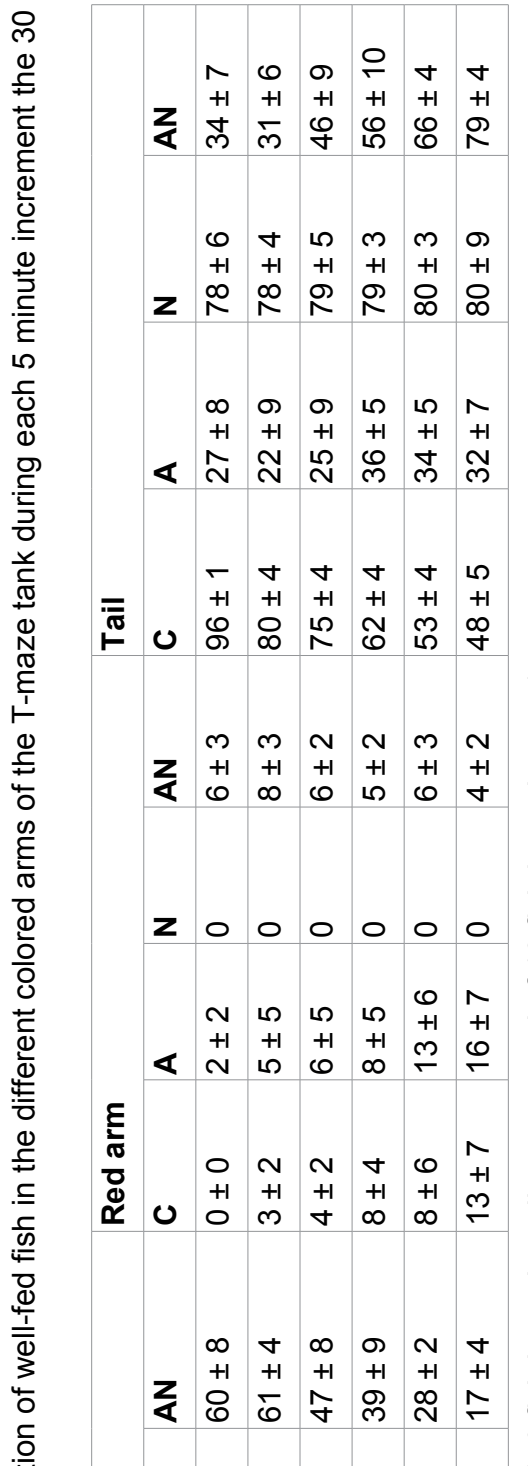

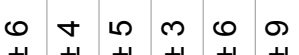

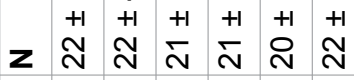

$\infty \wedge a 0$

$\begin{array}{lllllllll}2 & +1 & +1 & +1 & +1 & +1\end{array}$

$\varangle \nwarrow \Re$ \&

- $\infty \pi \infty \omega$

$\begin{array}{lllllll}0 & +1 & +1 & +1 & +1 & +1\end{array}$

৩ $\stackrel{+}{\sim} \bar{\sim}$ 尺े

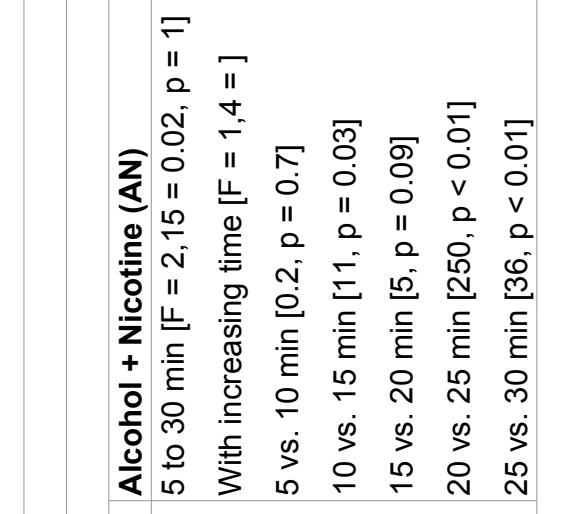

す্ণ

II II

อ

$\stackrel{2}{2}$

$6 \infty \pi$

॥

L

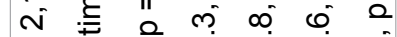

II

乙先

ป E

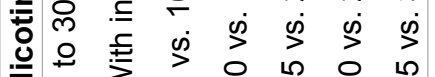
Z

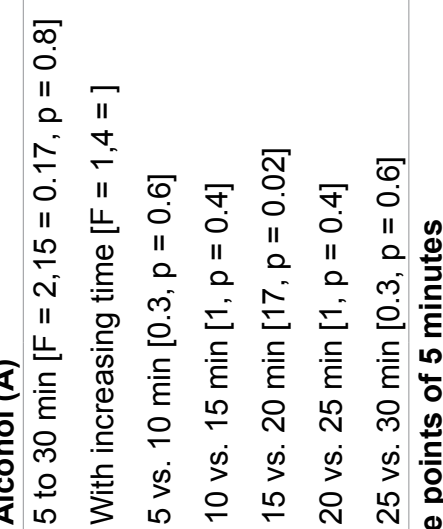

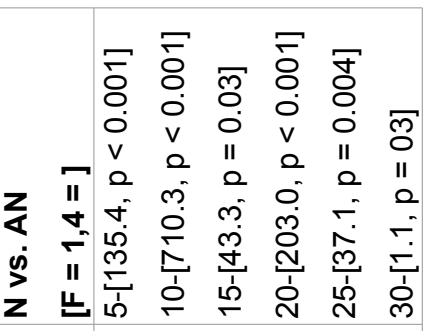
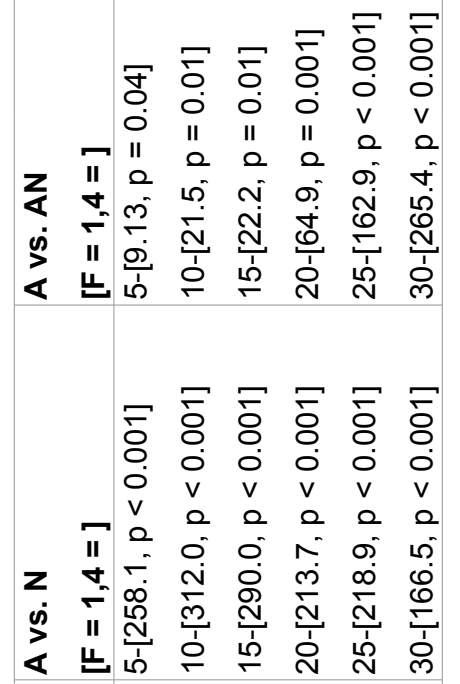

II II

ชิ

○. II

II

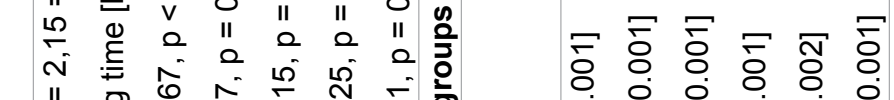
(2) 


\section{Experiment set III}

Screening of T-maze swimming pattern of starved shoaling Zebrafish. Shoaling fish maintained in a group of 6 were starved overnight before the onset of the experiment. Single fish were removed from the shoaling group and placed directly into the T-maze tank. Swimming pattern was then recorded. The initial 5 minutes of recording produced almost consistent type 3 swimming activity. This swimming pattern was neither affected by the repetition of the same experiment up to 6 consecutive days nor by placing food in either arm. Comparison of the total Percentage Fish Distribution (PDF) in the colored arms (green + red) at day $1(18.9 \pm 5.9 \%)$ and day $6(15.5 \pm 3.8 \%)$ showed $\mathrm{F}=1,10=1.41, \mathrm{p}=0.3)$. There was a lack of food seeking activity and no bias towards a color. In contrast, placing all 6 starved shoaling fish into the T-maze, produced type 3 swimming pattern in day one (PDF in the green arm $21.1 \pm 2.5 \%$ and red $\operatorname{arm} 26.1 \pm 2.1 \%, \mathrm{~N}=3$ replicate, 6 fish in each replicate], which progressively shifted to type 1 swimming pattern after subsequent days of recording when no food source was present. At day 5 and 6 , the swimming patterns were completely type 1 (PDF in green arm $53 \pm 2.8 \%, \mathrm{~N}=3$, 6 fish in each replicate). However, if the food was placed in the red arm of the T-maze, the swimming pattern shifted from type 3 to type 2 swimming pattern slowly (PDF in red arm $36.8 \pm 2.4 \%, \mathrm{~N}=3,6$ fish in each replicate). These results, clearly indicate that the removal of fish from their shoaling environment tremendously increase their anxiety levels. Consequently, even when isolated fish were hungry, they displayed no food seeking activity or color preference. In contrast, if the fish were housed individually for a few days before being exposed to the T-maze, they are largely confined to the arm of their preferred color. They also showed a reduction in food seeking activity in the non-preferred arm. Hence, shoaling seems to reduce the individual anxiety level of each fish and helps to enhance food seeking activity in the non-preferred color arm.

\section{Experiment set IV}

Swimming pattern produced by well-fed shoaling fish in the T-Maze after application of an aversive stimulus in the green arm. Shoaling fish when exposed to the T-maze displayed predominant movement toward the green arm during the first 10 minutes. With increasing time, they started moving slowly toward the red arm (Table 2 green arm column 2, and red arm column 1). This indicated that shoaling fish have their first place of preference in the green arm. However, with increasing time they began to explore the other regions of the T-maze. Three days conditioning training of the shoaling fish by the inclusion of strong water vibrations in the green arm, followed by observation of the swimming pattern for three days produced different results. The results showed type 4 swimming pattern (PDF in the tail $90.9 \pm 0.5 \%$, green arm $0.92 \pm 0.8 \%)$ with slow transition to type 3 swimming pattern (PDF in tail $37 \pm 13 \%$, green arm $23.6 \pm$ $6.7 \%$ and red arm $39.5 \pm 6.8 \%$ ), but not type 2 swimming pattern. These results clearly indicated that Zebrafish color preference can be modified by three days conditioning training to avoid green arm but not eliminated, as there was a significant PDF in the green arm after withdrawal from stimulation.

\section{Experiment set V}

Evaluation of the effect of addictive drugs on the color preference of well-fed shoaling Zebrafish. Shoaling fish (6 fish in a group) were subjected to either $1 \%$ alcohol $(\mathrm{v} / \mathrm{v})$ or $1 \mathrm{mg} / \mathrm{l}$ nicotine or a combination of both (mix alcohol $1 \% \mathrm{v} / \mathrm{v}$ and nicotine $1 \mathrm{mg} / \mathrm{l}$ ) for 30 minutes. Fish were then transferred to the holding area of the T-maze for 5 minutes after which the fish were released and swimming pattern recorded. This experiment was repeated for three more consecutive days with the same group of fish. Analysis of the total percentage fish distribution for the control, during the 30 minutes of recording showed that in the first day, fish preferentially shuttled between the tail $(65 \%)$ and the green arm (34\%). The total PDF in the red arm, however increased from $1 \%$ in the first day to $14 \%$ in the second day and $23 \%$ in the third day $(\mathrm{F}=2,6=89.2, \mathrm{p}$ $<0.001$ ) without affecting their distribution in green arm $(\mathrm{F}=2,6=3.9, \mathrm{p}=0.1)$. In other words, shoaling fish progressively shifted from type 1 to type 3 , but not type 2 . Pre-treatment of shoaling fish with alcohol or nicotine or co-treatment with both alcohol and nicotine, produced a drastic change in their distribution in the T-maze. The total PDF during the second day of recording in the green arm for alcohol, nicotine and alcohol nicotine co-treated fish were $63 \pm 2.6 \%, 22 \pm 1.6 \%$ and $49.6 \pm 2.0 \%$ respectively. Compared to the second day recording of control, the alcohol, nicotine and alcohol nicotine co-treated fish displayed a significant change in their green arm distribution (alcohol, $\mathrm{F}=1,4=206.9, \mathrm{P}<0.01$; Nicotine, $\mathrm{F}=1,4=$ 56.3, $\mathrm{P}=<0.01$; alcohol plus nicotine, $\mathrm{F}=1,4=57.4, \mathrm{p}<$ $0.01)$. However, these differences are minimal compared to the control on the third day with the same dosage of addictive drugs ( $p=0.4$ to 0.6 ). In contrast, PDF in the red arm remained below $20 \%$ in all the treatment groups throughout the three consecutive days of recording. This may be because of increased tolerance towards the same dosage of addictive drugs with increasing days of treatment. This trend continued into the fourth day of recording (data not included).

Since the first day of addictive drug treatment resulted in distinct differences in color preference, three replicates of each treatment (six fish per group) were performed. Analysis of PDF in the T-maze with pro- 
gressive 5 minutes of recording up to 30 minutes were summarized in (Table 2). As can be seen from (Table 2), control fish showed a progressive increase of PDF in the green arm with each 5 minute increment of recording. In contrast, alcohol treated fish showed $71 \pm 8 \%$ PDF in the green arm for the first 5 minutes and decreased slowly with each 5 minute increment of recording. However, the alcohol treated fish showed $52 \pm 5 \%$ PDF in the green arm during the last 5 minutes of recording (i.e., between 25-30 minutes) which was a much higher value compared to the control $39 \pm 6 \%$. The PDF in the red arm also increased during this period in both the control and alcohol treated groups, indicating that in both groups, the fish developed a tendency to shift from type 1 to type 3 swimming pattern. In contrast, nicotine treated fish showed around 20\% PDF in the green arm and maintained the same distribution throughout the 5 minutes incremental recording. There were no observable PDF in the red arm. Alcohol-nicotine co-treated fish on the other hand, produced alcohol-type PDF during the first 5 minutes of recording but rapidly decreased with each 5 minute increment of recording. During the last five minutes of recording, the swimming pattern was similar to the nicotine treated fish. This was interesting because both drugs have been reported to activate a common final neural pathway of the dopaminergic system which mediates the pleasurable feelings of reward despite their opposite molecular mode of action [18,19]. Our results clearly indicated that both drugs produced drastic changes in Zebrafish color preference during the initial days of exposure. Therefore, during the initial exposure, the effect of these drugs, even though they might be short lived, might not act the same on the neural pathway of the reward system. However, it seems that repeated exposure to these drugs is required for activation of the reward pathway. The repeated exposure to these drugs, are known to modify gene expression profiles [20-22]. Since both drugs produced different behaviors during initial exposure, the understanding of the molecular pathway of how these two drugs converse to activate the same reward system is not clear. But, the results obtained for the combined drugs, clearly indicated that alcohol can block the nicotine effect but the nicotine effect is able to overtake. This observation is in good agreement with earlier observations made by $[23,24]$. It should be noted that alcohol induced in toxication and learning impairments can be slashed down by nicotine intake $[25,26]$. However, our observation of complete swing from alcohol dependent behavior to nicotine dependent behavior after withdrawal from co-treatment within a short span of time cannot be considered as normal behavior. More in-depth studies are needed with different other behavior as well as biochemical parameters to validate co-addiction impact on heath and behavior.

\section{Conclusion}

Initial exposure of individually housed starved Zebrafish to the T-maze with colored arms produced four types of swimming patterns, that is, preferential shuttling between white tail and green arm; white tail and red arm; non-biased shuttling in both green and red arms and maximum retention in the white tail. However, subsequent exposure to the $\mathrm{T}$-maze showed an increase in the occurrence of the swimming pattern which was characterized by preferential shuttling between the white tail and green arm. This tendency could not be modified by starvation or by placing food in the red arm. In contrast, removing individually starved fish from their shoaling condition lead to increased anxiety levels and these fish showed no biased color preference between green and red and no food seeking activity. Shoaling starved fish on the other hand, have a greater preference for the green arm than the red arm, but they also showed an increase tendency of exploring the red arm and increased feeding activity within this arm. Well-fed shoaling fish showed preferential shuttling between the white tail and green arm with little exploration activity in the red arm. This preferential selection of the green colored arm by the well-fed shoaling fish can be modified but not eliminated by various stimuli such as aversive method sand addictive drugs. Overall our results reconfirmed previous observations made by [7], that Zebrafish have a greater preference for green than red and observations by Spence and Smith 2008, that natural color preferences can be modified but cannot be eliminated.

\section{Acknowledgements}

We are thankful to Dr. Paul Brown and Dr. Chukwuemeka R Nwokocha from the Department of Basic Medical Science, University of the West Indies Mona campus, Kingston Jamaica, for their valuable encouragements during experimentation process.

\section{Funding Information}

We gratefully acknowledge the financial support provided by the University of the West Indies Mona campus, Kingston Jamaica, in the form of a New Initiative Research Grant for the project entitled "Screening of nicotine and alcohol induced neurobehavioral interaction in co-addicted individuals using Zebrafish as an animal model" awarded to Dr. Mohammad K. Ali and the Graduate Studies Research grant awarded to Dawn Salmon.

\section{Author's Contribution}

Both authors equally contributed to the experiment designing, data analysis and manuscript preparation; while Dawn Salmon carried out all of the experiments in the Department of Basic Medical Sciences, University of the West Indies Mona campus, Kingston Jamaica. 
Citation: Salmon D, Ali MK (2017) An Assessment of Zebrafish Natural Color Preference and its Modification by Stimuli. J Mood Disord Ther 1(1):17-23

\section{Ethics}

This research was conducted after approval by the Research Ethics Committee of the University of the West Indies Mona campus, Kingston, Jamaica.

\section{References}

1. Stewart A, Wong K, Cachat J, et al. (2011) Zebrafish models to study drug abuse-related phenotypes. Rev Neurosc 22: 95-105.

2. Levin ED (2011) Zebrafish assessment of cognitive improvement and anxiolysis: filling the gap between in vitro and rodent models for drug development. Rev Neurosci 22: 75-84.

3. Piccinetti CC, Migliarini B, Olivotto I, et al. (2010) Appetite regulation: the central role of melatonin in Danio rerio. Horm Behav 58: 780-785.

4. Piccinetti CC, Migliarini B, Olivotto I, et al. (2013) Melatonin and peripheral circuitries: insights on appetite and metabolism in danio rerio. Zebrafish 10: 275-282.

5. Gerlai R (2011) Associative learning in Zebrafish (Danio rerio). Methods Cell Biol 101: 249-270.

6. Sison M, Gerlai R (2011) Associative learning performance is impaired in zebrafish (Danio rerio) by the NMDA-R antagonist MK-801. Neurobiol Learn Mem 96: 230-237.

7. Oliveira J, Silveira M, Chacon D, et al. (2015) The zebrafish world of colors and shapes: preference and discrimination. Zebrafish 12: 166-173.

8. Moorman SJ (2001) Development of Sensory Systems in Zebrafish (Donio rerio). ILAR J 42: 292-298.

9. Levin ED, Chen E (2004) Nicotinic involvement in memory function in zebrafish. Neurotoxicol Teratol 26: 731-735.

10. Blaser RE, Penalosa YM (2011) Stimuli affecting zebrafish (Danio rerio) behavior in the light/dark preference test. Physiol Behav 104: 831-837.

11. Colwill RM, Raymond MP, Ferreira L, et al. (2005) Visual discrimination learning in zebrafish (Danio rerio). Behav Process 70: 19-31.

12. Avdesh A, Martin-Iverson MT, Mondal A, et al. (2012) Evaluation of color preference in zebrafish for learning and memory. J Alzheimer's Dis 28: 459-469.

13. Spence R, Smith C (2008) Innate and learned colour preference in the zebrafish, Danio rerio. Ethol 114: 582-588.
14. Miller N, Greene K, Dydinski A, et al. (2013) Effects of nicotine and alcohol on zebrafish (Danio rerio) shoaling. Behav Brain Res 240: 192-196.

15. Wu J, Gao M, Taylor DH, et al. (2014) Neuronal nicotinic acetylcholine receptors are important targets for alcohol reward and dependence. Acta Pharmacol Sin 35: 311-315.

16. Rahman S, Engleman EA, Bell RL, et al. (2016) Recent advances in nicotinic receptor. signaling in alcohol abuse and alcoholism. Prog Mol Biol Trans Sci 137: 183-201.

17. Castranova D, Lawton A, Lawrence C, et al. (2011) The Effect of Stocking Densities on Reproductive Performance in Laboratory Zebrafish (Danio rerio). Zebrafish 8: 141-146.

18. Schlaepfer IR, Hoft NR, Ehringer MA, et al. (2008) The genetic components of alcohol and nicotine co-addiction: from genes to behavior. Curr Drug Abuse Rev 1: 124-134.

19. Doyon WM, Thomas AM, Ostroumov A, et al. (2013) Potential substrates for nicotine and alcohol interactions: a focus on the mesocorticolimbic dopamine system. Biochem Pharmacol 86: 1181-1193.

20. Kily LJ, Cowe YC, Hussain O, et al. (2008) Gene expression changes in a zebrafish model of drug dependency suggest conservation of neuro-adaptation pathways. J Exp Biol 211: 1623-1634.

21. Sureshchandra S, Rais M, Stull C, et al. (2016) Transcriptome profiling reveals disruption of innate immunity in chronic heavy ethanol consuming female rhesus macaques. PloS One 11: e0159295.

22. Kanungo J, Cuevas E, Guo X, et al. (2012) Nicotine alters the expression of molecular markers of endocrine disruption in zebrafish. Neurosci Lett 526: 133-137.

23. Korkosz A, Taracha E, Plaznik A, et al. (2005) Extended blockade of the discriminative stimulus effects of nicotine with low doses of ethanol. Eur J Pharmacol 512: 165-172.

24. Ali MK, Ragoobirsingh D (2013) Nicotine-alcohol induced differential feeding behaviour. OA Alcohol 1: 14.

25. Parnell SE, West JR, Chen WJ, et al. (2006) Nicotine decreases blood alcohol concentrations in adult rats: A phenomenon potentially related to gastric function. Alcohol Clin Exp Res 30: 1408-1413.

26. Gulick D, Gould TJ (2011) Nicotine acts in the anterior cingulate, but not dorsal or ventral hippocampus, to reverse ethanol-induced learning impairments in the plus-maze discriminative avoidance task. Addict Biol 16: 176-188. 